\title{
Conformational Analysis of the Sodium/12-Crown-4 Complex: Photoionization and ab Initio Molecular Orbital Studies
}

\author{
T.-S. Yeh and T.-M. Su* \\ Department of Chemistry, National Taiwan University, Taipei, Taiwan, and The Institute of the Atomic and \\ Molecular Sciences, Academia Sinica, Taipei, Taiwan
}

Received: March 17, 1998; In Final Form: April 24, 1998

\begin{abstract}
The molecular complexes formed between sodium atoms and 12-crown-4 molecules were generated in a flow reactor and studied with photoionization mass spectroscopy. The photoionization threshold energies of these complexes, which are dependent on the complex conformations, were obtained from the analysis of the photoionization efficiency spectra. Ab initio molecular orbital calculations were performed on the possible conformers of these complexes and their corresponding ionic states. With the help of theoretical calculations, the photoionization threshold energy of the $C_{4}$ conformer of $\mathrm{Na}$ (12-crown-4) was determined to be $3.01 \pm$ $0.02 \mathrm{eV}$, and the $C_{s}$ conformer to be $3.36 \pm 0.04 \mathrm{eV}$. The photoionization threshold energy of the $C_{i}$ conformer was estimated to be $3.82 \pm 0.04 \mathrm{eV}$. The conformation-dependent valence electron spatial distributions, bond dissociation energies, and photoionization threshold energies of the $\mathrm{Na}$ (12-crown-4) complex could be rationalized and classified in the framework of the general properties of the complexes formed between the sodium atoms and Lewis base molecules.
\end{abstract}

\section{Introduction}

Since the first successful synthesis of an alkalide in 1973, more than 40 alkalides and 5 electrides have been synthesized and characterized by Dye and co-workers. ${ }^{1-3}$ Experimentally, these compounds have been shown to have a wide range of interesting structures and properties. Theoretically, because of the comparatively large number of atoms per unit cell for these systems, reliable solutions were difficult to obtain by ab initio methods. It was only quite recently that some simpler electride systems were studied theoretically and the nature of the valence electrons was elaborated. ${ }^{4-8}$

In the past few years, molecular complexes formed between the alkali metal atoms and multifunctional Lewis bases, such as ethylenediamine, 1,2-ethanediol, and 1,2-dimethoxyethane, have been studied in this laboratory. ${ }^{9-11}$ The conformationdependent bond dissociation energies and photoionization threshold energies of these complexes were emphasized in these works. In the present study, we extend the Lewis base molecules to cyclic crown ethers and, more specifically, 12crown-4 (12C4). Their molecular complexes with the alkali metal atoms may be regarded as the first intermediates in the formation process of the corresponding electrides and alkalides. Additionally, these complexes are also simple enough to be treatable by higher level ab initio methods. In the present study the photoionization efficiency spectrum of $\mathrm{Na}(12 \mathrm{C} 4)$ was taken. Its conformation-dependent Mulliken electron population, ionization energies, and bond dissociation energies were calculated by the ab initio methods. With the help of the theoretical results, the measured ionization threshold energies were then assigned as the ionization threshold energies of specific complex conformers populated by the thermal energy. The present study might shed some light on the general behavior of valence electrons in electrides and alkalides.

Since both the neutral and ionic states of the complexes are involved in the present one-photon ionization process, the conformation-dependent geometrical structures and bond energies of $\mathrm{Na}^{+}(12 \mathrm{C} 4)$ calculated by the ab initio methods were also reported. As there were already quite a few theoretical and experimental works on the alkali metal ion/crown ether complexes in the literature, ${ }^{12-16}$ in light of the present study, a general discussion on the bonding energies of the ionic complexes was also given.

\section{Experimental Section}

The details of the experimental arrangement, which is basically composed of a photoionization mass spectrometer and a flow reactor, have been reported in previous publications. ${ }^{9-11,17}$ Only a brief account of the relevant experimental conditions for the present molecular complexes is given here.

The sodium vapor was first generated by heating a sodium oven to about $585 \mathrm{~K}$, and then it was introduced into the flow reactor by a minor argon gas flow of about 0.5 Torr partial pressure. The temperature of the sodium flow tube was kept at $513 \mathrm{~K}$. In previous photoionization experiments, the Lewis base molecules, such as ethylenediamine and 1,2-dimethoxyethane, all have high enough vapor pressures to form a manageable vapor flow at temperatures around $370 \mathrm{~K}$ or below. Their vapors were fed into the main flow reactor through an outside sample holder kept at a fixed temperature by a thermostat. Because of the relatively low vapor pressure of $12 \mathrm{C} 4$, in the present experiment, the sample holder was placed directly in the main flow chamber and kept at the same temperature. The photoionization efficiency spectra of the monosodium complex were taken with a main reactor temperature of $328 \mathrm{~K}$ and a $12 \mathrm{C} 4$ vapor pressure of around 0.05 Torr. The total pressure of the flow reactor was kept at around 2.4 Torr by an argon buffer flow.

\section{Ab Initio Molecular Orbital Calculations}

The molecular orbital calculations were carried out with the Gaussian 94 package. ${ }^{18}$ The geometric parameters and the 
TABLE 1: Relative Conformation Energies (eV) of 12-Crown-4 ${ }^{a}$

\begin{tabular}{lcccc}
\hline & $C_{4}$ & $C_{s}$ & $C_{i}$ & $S_{4}$ \\
\hline HF/6-311+G** & 0.300 & 0.139 & 0.076 & 0 \\
MP2/6-31+G* & 0.345 & 0.194 & 0.112 & 0 \\
MP2/6-311+G** & 0.287 & 0.152 & 0.092 & 0 \\
B3PW91/6-311+G** & 0.236 & 0.113 & 0.069 & 0 \\
B3LYP/6-311+G** & 0.258 & 0.126 & 0.076 & 0
\end{tabular}

${ }^{a} \mathrm{HF} / 6-31 \mathrm{G}^{*}$ optimized geometry.

harmonic vibrational frequencies of the neutral and ionic molecular complexes were calculated at the HF/6-31G* level. The single-point energies were then calculated up to the $\mathrm{MP} 2(\mathrm{Fu}) / 6-311+\mathrm{G}^{* *} / / \mathrm{HF} / 6-31 \mathrm{G}^{*}$ and B3PW91/6-311+G**// $\mathrm{HF} / 6-31 \mathrm{G}^{*}$ levels. The adequacy of these calculation levels shall be discussed in the following results section.

The conformation notation of 12-crown-4 is in accord with those reported in the literature. ${ }^{15,19}$ The conformation-dependent vertical and adiabatic ionization energies of the neutral complexes and the sudden and adiabatic equilibrium bond dissociation energies of both the neutral and ionic complexes were reported. All the theoretical bond dissociation energies were corrected with basis set superposition errors calculated by the counterpoise method.

\section{Results and Discussion}

The results are discussed in the following six subsections.

4.A. Basic Four Conformers of 12-Crown-4 and an Evaluation of Various Calculation Levels. Four basic conformers of the free 12-crown-4 molecule, which are labeled $C_{4}$, $C_{s}, C_{i}$, and $S_{4}$ according to their geometric symmetries, are considered in this report. Upon complexation with the sodium atom, these four conformers are capable of forming their own stable and unique tetra-, tri-, di-, and monodentate sodium/12C4 complexes according to the above symmetric order. As shown in Table 1, at the present ab initio calculation levels, the relative energies of these four conformers also follow the same energy ordering in which the most stable conformer is in $S_{4}$ symmetry. These values are in general agreement with those of similar calculation levels reported in the literature: the RHF/DZ+P energies of Seidl and Schaefer; ${ }^{19}$ and the RHF, MP2, BLYP, and BP86 energies at the $6-31+\mathrm{G}^{*}$ basis set by Feller et al. ${ }^{15}$ Among the present calculation results, the relative conformation energies of B3LYP are in good agreement with those of B3PW91. These values, in turn, are all slightly lower than the corresponding MP2 energies by about $0.026 \mathrm{eV}$. It has been suggested that, for a more accurate prediction of the conformational energies of poly(ethylene oxide), at least, moderately large basis sets and correlation energy recovery are required. ${ }^{15,20}$ The good agreement of the $C_{i}$ conformation energy of 0.092 $\mathrm{eV}$ calculated at the MP2/6-311+G** level with that of 0.082 $\mathrm{eV}$ calculated at the MP2/aug-cc-pVDZ//MP2/cc-pVDZ level by Feller et al. ${ }^{15}$ also supports this general observation. The present relative MP2 conformational energies of the four basic conformers are estimated to be accurate to within $0.026 \mathrm{eV}-$ the energy difference between the MP2 and B3LYP energiesand are reckoned to be adequate for the present purpose.

Table 2 shows the sudden dissociation energies of the $\mathrm{Na}$ $\left(C_{4}\right)$ and $\mathrm{Na}^{+}\left(C_{4}\right)$ conformers with BSSE corrections obtained at various calculation methods and levels. Four general observations can be drawn from these energies. First, the electron correlation is more pronounced in the ionic state than the neutral state. Second, with the present highest $6-311+\mathrm{G}^{* *}$ basis set, the dissociation energies are generally in quite good
TABLE 2: Sudden Equilibrium Bond Dissociation Energies $(\mathrm{eV})$ of $\mathrm{Na}(12 \mathrm{C} 4)$ and $\mathrm{Na}^{+}(12 \mathrm{C} 4)$ in $C_{4}$ Conformation at Various Calculation Levels ${ }^{a}$

\begin{tabular}{lccc}
\hline & geometry & $\begin{array}{c}\mathrm{Na}\left(C_{4}\right) \rightarrow \\
\mathrm{Na}+C_{4}\end{array}$ & $\begin{array}{c}\mathrm{Na}^{+}\left(C_{4}\right) \rightarrow \\
\mathrm{Na}^{+}+C_{4}\end{array}$ \\
\hline $\mathrm{HF} / 6-311+\mathrm{G}^{* *}$ & $\mathrm{HF}^{b}$ & 0.589 & 3.185 \\
MP2/6-31+G* & $\mathrm{HF}$ & 0.635 & 3.054 \\
MP2/6-311+G** & $\mathrm{HF}$ & 0.586 & 2.949 \\
B3PW91/6-311+G** & $\mathrm{HF}$ & 0.487 & 2.979 \\
B3LYP/6-311+G** & $\mathrm{HF}$ & 0.491 & 3.081 \\
MP2/6-311+G** & MP2 & 0.576 & 2.965 \\
B3LYP/6-311+G** & MP2 & 0.506 & 3.097
\end{tabular}

${ }^{a}$ All energies are BSSE corrected. ${ }^{b} \mathrm{HF} / 6-31 \mathrm{G}^{*}$ optimized geometry. MP2/6-31G* optimized geometry.

agreement with each other among the three calculation methods of MP2, B3PW91, and B3LYP. Third, at a closer examination, for the neutral complex, the dissociation energies of B3PW91 and B3LYP are essentially the same, and both are less than the MP2 energy by about $0.10 \mathrm{eV}$. For the ionic complex, the MP2 and B3PW91 dissociation energies are essentially identical, and both are less than the B3LYP value by about $0.12 \mathrm{eV}$. Finally, the energy variation caused by the differences in the MP2- and HF-optimized geometries is only about $0.015 \mathrm{eV}$ for both the neutral and ionic complexes.

It has been found previously that, for the sodium-dimethoxyethane complexes and other related systems, the ionization energies could be predicted quite accurately by the B3PW91 method. ${ }^{11}$ Along with the above observations, the present $\mathrm{Na} /$ $12 \mathrm{C} 4$ and its ionic complexes shall be discussed mainly in terms of the theoretical results of the MP2 and B3PW91 methods at the HF geometries.

4.B. Geometric Structures of $\mathrm{Na}(12 \mathrm{C} 4)$ and $\mathrm{Na}^{+}(12 \mathrm{C4})$. As mentioned previously, each of the four basic $12 \mathrm{C} 4$ conformers could combine with the sodium atom to form its own most stable and also unique complex conformer. Figures 1 and 2 show the ball-and-stick representations of these conformers. The most stable $\mathrm{Na} / C_{4}$ complex is in a tetradentate conformation which still preserves the $C_{4}$ symmetry of the ligand. The most stable $\mathrm{Na} / C_{s}$ complex is in a tridentate conformation and also still in the $C_{s}$ symmetry. Similarly the most stable $\mathrm{Na} / C_{i}$ complex is in a bidentate conformation, but its ligand's symmetry is already distorted and not in a strict $C_{i}$ form. Finally, only a monodentate conformation is possible for the most stable $\mathrm{Na} / S_{4}$ complex, and its $12 \mathrm{C} 4$ symmetry is also in a distorted $S_{4}$ form. In this report, the symbols $C_{i}$ and $S_{4}$ are still used for the distorted $\mathrm{Na} / C_{i}$ and $\mathrm{Na} / S_{4}$ complexes, respectively. The corresponding conformation notations for the above specific complexes are denoted as $\mathrm{Na}\left(C_{4}\right), \mathrm{Na}\left(C_{s}\right), \mathrm{Na}\left(C_{i}\right)$, and $\mathrm{Na}\left(S_{4}\right)$, respectively.

In the case of the $\mathrm{Na}^{+} / 12 \mathrm{C} 4$ complex, among the four basic ligand conformations, only three locally stable conformers are located: $\mathrm{Na}^{+}\left(C_{4}\right), \mathrm{Na}^{+}\left(C_{s}\right)$, and $\mathrm{Na}^{+}\left(S_{4}\right)$. The $\mathrm{Na}^{+}\left(C_{i}\right)$ conformer is not locally stable at the HF/6-31+G* level. Being similar to their neutral counterparts, the first two cationic conformers preserve their symmetries upon complexation. Nevertheless, owing to the strong ion-dipole interaction, the third $S_{4}$ conformer actually settles down to a $C_{2}$ symmetry form, in which the sodium cation is sitting on the center of the ligand as in the case of the $C_{4}$ conformer but with two different $\mathrm{Na}^{+}-\mathrm{O}$ bond distances.

For the geometric structures of the neutral complex, the $12 \mathrm{C} 4$ ligand is essentially in the same geometries as the free state in all four conformations. The shortest sodium-oxygen bond distances are $2.559,2.506,2.454$, and $2.446 \AA$ for the $C_{4}, C_{s}$, 


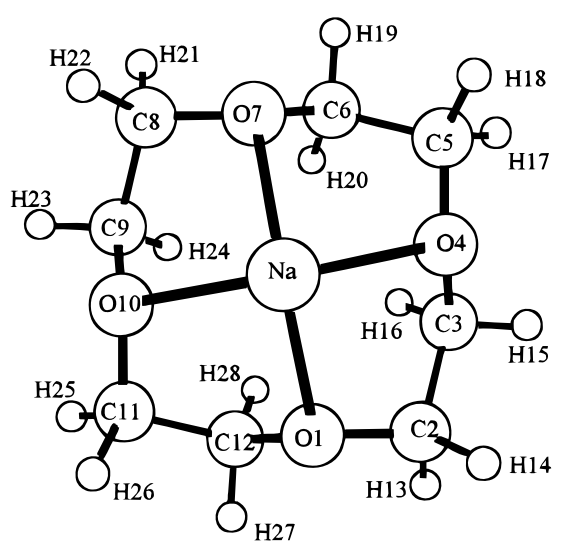

$\mathrm{Na}(12 \mathrm{C} 4) \mathrm{C}_{4}$ Conformer

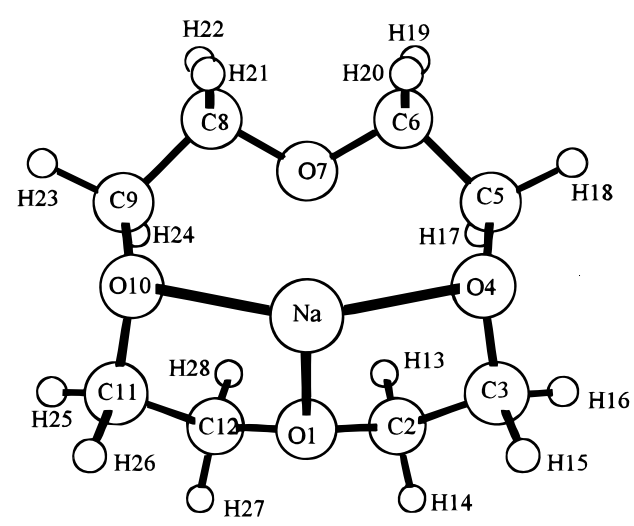

$\mathrm{Na}(12 \mathrm{C} 4) \mathrm{C}_{\mathrm{s}}$ Conformer

Figure 1. Ball-and-stick representations of the $C_{4}$ and $C_{s}$ conformers of $\mathrm{Na}(12 \mathrm{C} 4)$.

$C_{i}$, and $S_{4}$ conformers, respectively. The decreasing of the bond lengths is in good correlation with the increasing of the sodium coordination number of the conformers. In the case of the cationic complexes, the shortest $\mathrm{Na}^{+}-\mathrm{O}$ bond distances of $\mathrm{Na}^{+}$$\left(C_{4}\right)$ and $\mathrm{Na}^{+}\left(C_{s}\right)$ are 2.332 and $2.274 \AA$, respectively. They are shorter then the corresponding bond distances of the neutral counterparts by about $0.23 \AA$. The ligand geometry of the $C_{4}$ conformer is quite close to that of its corresponding neutral one. For the $C_{s}$ complex, compared with its neutral counterpart, there are moderate changes of the dihedral angles of the molecular fragments that are bonded to the oxygen atom located on the opposite side of the sodium ion. In the case of $\mathrm{Na}^{+}\left(C_{s}\right)$, owing to the strong ion-dipole interaction, there are substantial dihedral angle changes for those molecular fragments that are directly bonded to the two oxygen atoms located on the opposite side of the sodium ion. Its $\mathrm{Na}^{+}-\mathrm{O}$ distance is shorter than that of the corresponding neutral conformer by $0.16 \AA$. The detailed geometric parameters and harmonic vibrational frequencies of $12 \mathrm{C} 4, \mathrm{Na}(12 \mathrm{C} 4)$, and $\mathrm{Na}^{+}(12 \mathrm{C} 4)$ discussed in this report are tabulated in the Supporting Information.

4.C. Mulliken Population Analysis and Bonding Nature of $\mathrm{Na}(12 \mathrm{C} 4)$ Conformers. In a study of $\operatorname{Li}(9 \mathrm{C} 3)_{2}$ with the ligands in the form of hexadentate $D_{3 d}$ symmetry, Rencsok et al. have shown that the valence electron is predominantly in a Rydberg-type, loosely bound-to-the-complex state., ${ }^{5,6}$ More recently, Hashimoto and Morokuma studied the stability and

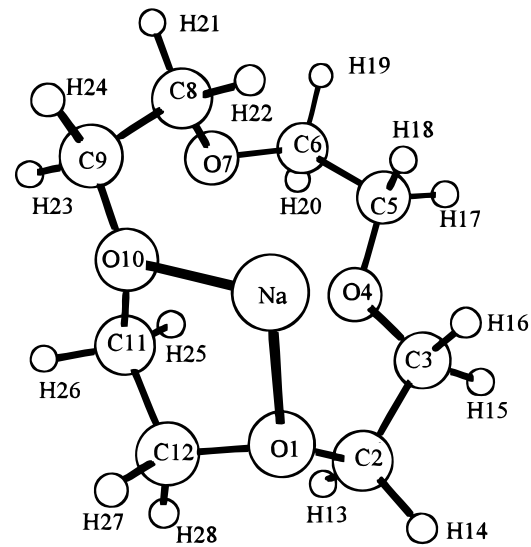

$\mathrm{Na}(12 \mathrm{C} 4) \mathrm{C}_{\mathrm{i}}$ Conformer

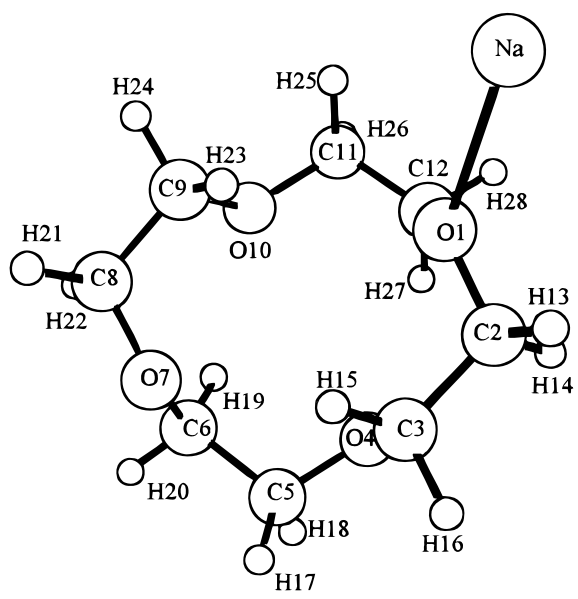

$\mathrm{Na}(12 \mathrm{C} 4) \mathrm{S}_{4}$ Conformer

Figure 2. Ball-and-stick representations of the $C_{i}$ and $S_{4}$ conformers of $\mathrm{Na}(12 \mathrm{C} 4)$. See the text for the symbol definitions.

TABLE 3: Mulliken Gross Population of $\mathrm{Na}$ and $\mathrm{Na}(12 \mathrm{C4})$ in Na Valence sp Basis Functions ${ }^{a}$

\begin{tabular}{lccccc}
\hline & \multicolumn{4}{c}{ Na basis functions } & \\
\cline { 2 - 4 } conformer & $2 \mathrm{sp}$ & $3 \mathrm{sp}$ & $4 \mathrm{sp}$ & sum & Na charge \\
\hline $\mathrm{Na}$ & 0.50 & 0.51 & 0.01 & 1.02 & 0 \\
$\mathrm{Na}\left(S_{4}\right)$ & 0.45 & 0.64 & 0.03 & 1.12 & -0.09 \\
$\mathrm{Na}\left(C_{i}\right)$ & 0.38 & 0.73 & 0.06 & 1.17 & -0.16 \\
$\mathrm{Na}\left(C_{s}\right)$ & 0.35 & 0.74 & 0.13 & 1.22 & -0.20 \\
$\mathrm{Na}\left(C_{4}\right)$ & 0.30 & 0.61 & 0.17 & 1.08 & -0.06
\end{tabular}

${ }^{a}$ Calculated at the $\mathrm{HF} / 6-31+\mathrm{G}^{*}$ level.

structure of $\mathrm{Na}\left(\mathrm{H}_{2} \mathrm{O}\right)_{n}$ and $\mathrm{Na}\left(\mathrm{NH}_{3}\right)_{n}(n=1-6) .{ }^{21,22}$ Their Mulliken gross orbital population on sodium shows that the character of the extra electron varies from the normal valence electron to the diffuse, solvated-like electron as the ligand number $n$ increases from 1 to 6 , with $n=2,3$, and 4 falling between these two extremes. Along this general line, the conformation-dependent character of the sodium valence electron in $\mathrm{Na}(12 \mathrm{C} 4)$ shall be discussed.

Table 3 shows the Mulliken gross orbital population of the four $\mathrm{Na}(12 \mathrm{C} 4)$ conformers on the sodium valence $\mathrm{sp}$ basis functions and also the Mulliken sodium atomic charge at the $\mathrm{HF} / 6-31+\mathrm{G}^{*}$ level. Two general population trends were 

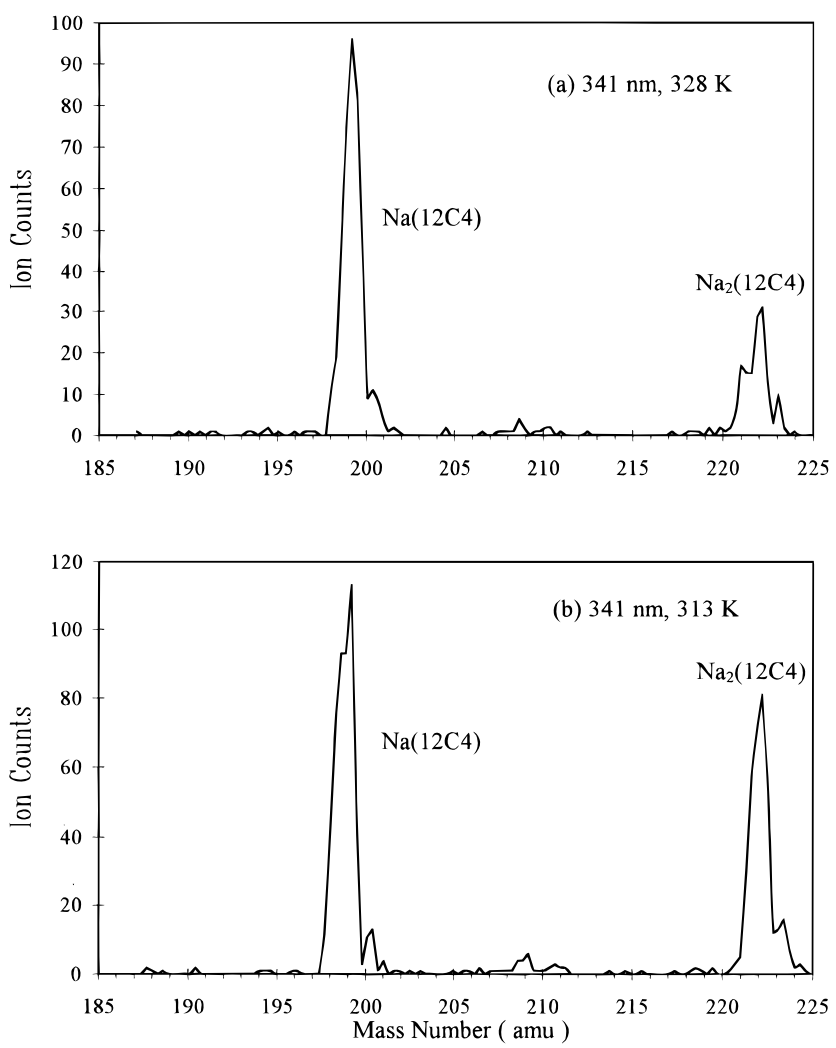

Figure 3. Photoionization mass spectra of $\mathrm{Na}(12 \mathrm{C} 4)$ at $341 \mathrm{~nm}$ laser radiation.

observed. First, the populations in the inner valence 2 sp basis functions decrease monotonically, while those in the diffusive $4 \mathrm{sp}$ basis functions increase monotonically, as the sodium atom varies from the free sodium state to the tetradentate $C_{4}$ conformation. Second, the populations in the outer valence $3 \mathrm{sp}$ basis functions, the summation populations in the complete valence sp basis functions, and the Mulliken sodium atomic charges all increase from the free sodium state to the tridentate $C_{s}$ conformation and then decrease quite substantially to the tetradentate $C_{4}$ conformation. The conformation-dependent electron populations of $\mathrm{Na}(12 \mathrm{C} 4)$ are consistent with the general characteristics of the valence electron in the $\mathrm{Na}\left(\mathrm{H}_{2} \mathrm{O}\right)_{n}$ and $\mathrm{Na}\left(\mathrm{NH}_{3}\right)_{n}$ clusters. In the mono- to tridentate conformations, both the charge transfer from the ligand to the sodium atom and the shift of the sodium inner valence population to the outer and diffusive valence basis functions suggest that the nature of the $\mathrm{Na}-\mathrm{O}$ bond still mainly belongs to the category of the localized interactions between the alkali metal atom and simple Lewis base molecules, a subject having been under intensive study for the past two decades or so. ${ }^{21-27}$ As the conformation reaches the tetradentate $C_{4}$ state, the abrupt decrease of both the sodium atomic charge and the inner and outer valence electron populations and the monotonic increase of the diffuse electron population suggest that the valence electron has acquired some character of the valence electron observed in the $\mathrm{Li}(9 \mathrm{C} 3)_{2}$ complex and the higher $\mathrm{Na}\left(\mathrm{NH}_{3}\right)_{n}$ clusters, i.e., diffusive, Rydberg-type, and solvated-like.

4.D. Photoionization Mass Spectra and Photoionization Efficiency Spectra of $\mathrm{Na}(\mathbf{1 2 C 4})$. Figure 3 shows the photoionization mass spectra of $\mathrm{Na}(12 \mathrm{C} 4)$ and $\mathrm{Na}_{2}(12 \mathrm{C} 4)$ with 341 $\mathrm{nm}$ photon radiation at 328 and $313 \mathrm{~K}$ flow reactor temperature, respectively. Both mass peaks would disappear if either the sodium or $12 \mathrm{C} 4$ vapor was terminated. The relative signal ratio of the disodium to monosodium complex depends on the flow reactor temperatures, sodium vapor pressure, and the ionization
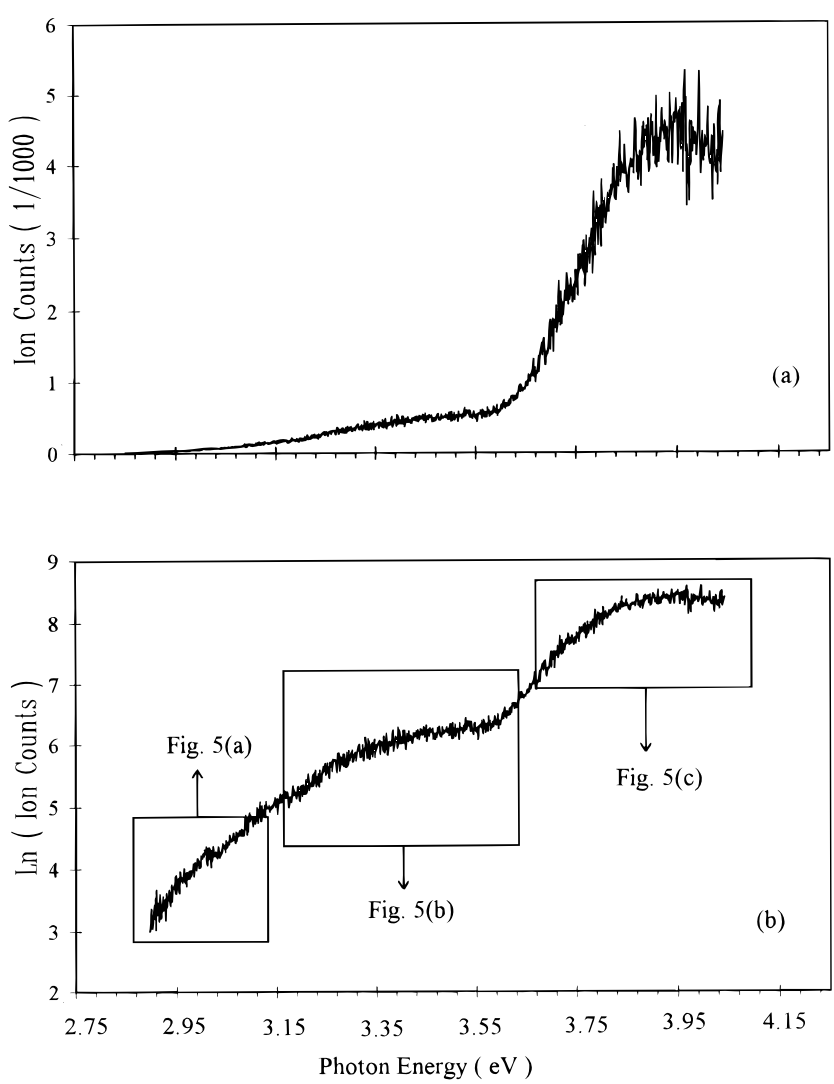

Figure 4. Photoionization efficiency spectrum of $\mathrm{Na}(12 \mathrm{C} 4)$ (a) and the corresponding Watanabe plot (b).

wavelength. To minimize the possible contamination of the monosodium complex signal by the photoionized disodium complex through the dissociation process in the higher photon energy regime, in the present experiment, the flow reactor was kept at $328 \mathrm{~K}$ and the sodium oven temperature was set at the lower side. Under such experimental conditions the disodium complex signal was determined to be less than $25 \%$ of the monosodium signal by setting the photon energies between threshold decomposition energy for the fragmentation of the disodium complex ions into the monosodium complex ions and sodium atoms and the threshold electron ionization energy. Figure 3 shows a typical example in this wavelength region. In other words, in the worst situation, at most $20 \%$ of the observed monosodium signal might come from the contribution of the disodium complex if the excess photon energy was higher than the required energy for the sodium atoms to split away from the ionic disodium complex and also all the disodium complex ions underwent the dissociation. ${ }^{28}$

The photoionization efficiency spectra of monosodium complexes and their corresponding Watanabe plot are shown in Figure 4. Three general steplike spectral features could be clearly identified, and their corresponding enlarged Watanabe plots are shown in Figure 5. The first and also the lowest ionization threshold was determined to be $3.01 \pm 0.02 \mathrm{eV}$, the second one to be $3.36 \pm 0.04 \mathrm{eV}$, and the third one to be 3.82 $\pm 0.04 \mathrm{eV}$. The quoted uncertainties were estimated from the uncertainties in the selection of the linear regions in the Watanabe plots and the intrinsic accuracy of the present experimental setup. Note that the threshold ionization energies of $\mathrm{Na}$ and $\mathrm{Na}_{2}$ were measured to be 5.134 and $4.875 \mathrm{eV}$ by the present method. Compared with the reported adiabatic ionization energies of $5.139 \mathrm{eV}^{29}$ and $4.895 \mathrm{eV},{ }^{30}$ respectively, the agreement is excellent. 

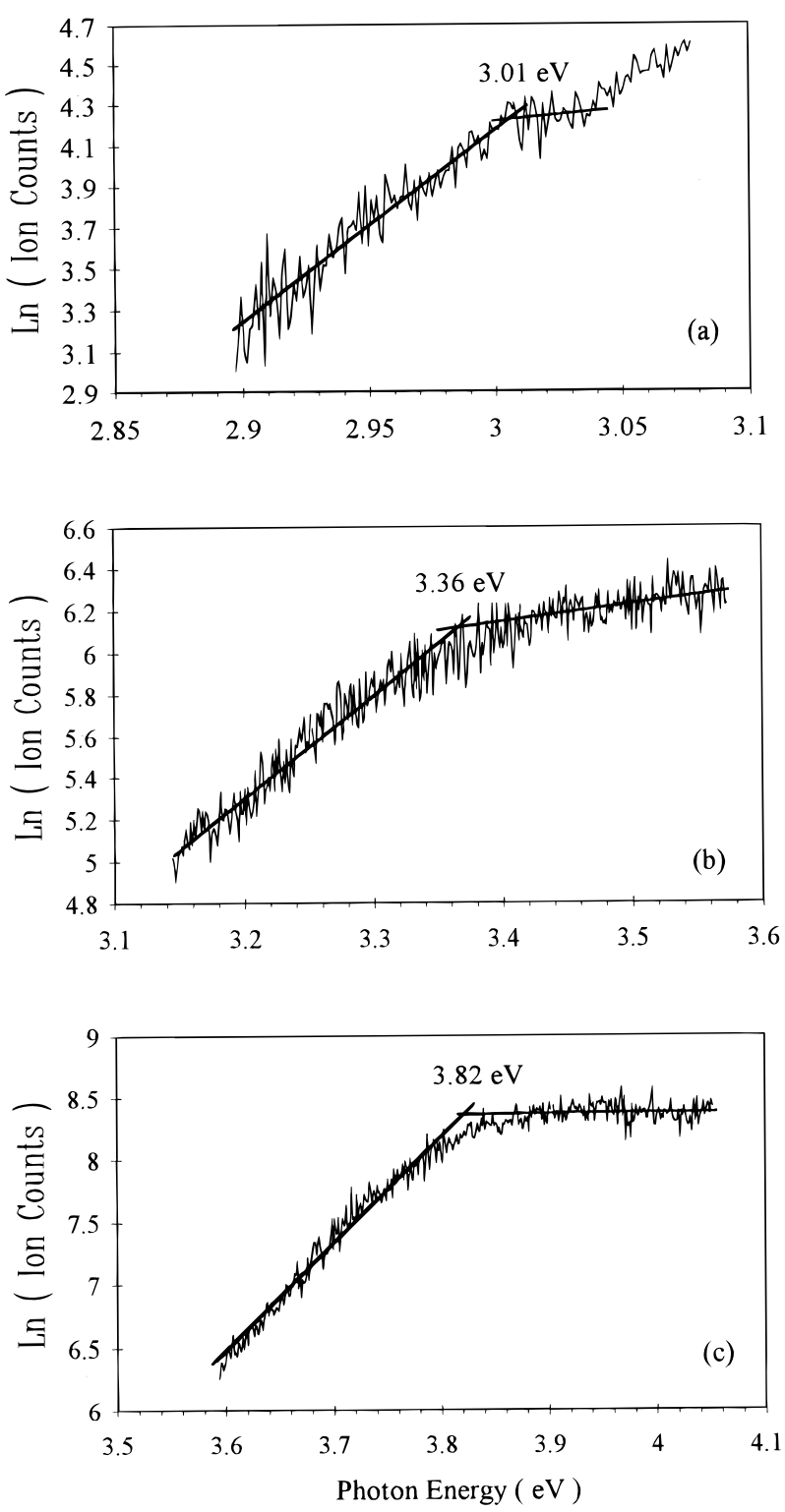

Figure 5. Enlarged Watanabe plot of Figure 4.

In the following section, with the help of the results of molecular orbital calculations, the three measured consecutive ionization threshold energies were assigned as the ionization energies of three specific conformers of $\mathrm{Na}(12 \mathrm{C} 4)$.

4.E. Comparisons between Theoretical and Experimental Results. Table 4 shows the theoretical vertical and adiabatic equilibrium ionization energies of the sodium atom and the four possible conformers of $\mathrm{Na}(12 \mathrm{C} 4)$ at the $\mathrm{HF}, \mathrm{MP} 2$, and B3PW91 levels. As the table shows, for the same ionization quantity, the HF method always yields the smallest ionization energy, followed consecutively by the MP2 and the B3PW91 methods. Recent studies of the $\mathrm{Na}(1,2$-dimethoxyethane) complexes and related systems suggested that, among the three calculation levels, the B3PW91 calculations yield the most accurate prediction on the ionization energies, followed by the MP2 and HF methods. ${ }^{11}$ The B3PW91 ionization energies shall be exclusively used in the present discussion. Those of the HF and MP2 methods are listed for comparison purpose.

The adiabatic ionization energies with respect to the most stable ionic conformer $\mathrm{Na}^{+}\left(C_{4}\right)$ of the $C_{4}, C_{s}$, and $C_{i}$ complexes are close to each other to within $0.02 \mathrm{eV}$, an indication that the neutral-state energies of these three conformers happen to be quite similar to each other. In other words, the $\mathrm{Na}-\mathrm{O}$ bonding strength is similar to the energy differences among the $12 \mathrm{C} 4$ conformations such that they almost completely counterbalance each other in the formation of the tetra-, tri-, and bidentate conformers. This accidental balance of the interaction strengths is also observed in the adiabatic bond dissociation energies of the neutral complexes discussed in the following section. There is appreciable change between the adiabatic ionization energy with respect to its original conformation state and the corresponding vertical ionization energy, especially for the $C_{s}, C_{i}$, and $S_{4}$ states, in which the $C_{i}$ ionic state is even not stable locally. These suggest that there are major equilibrium geometric changes between the neutral and ionic conformers, especially in the dihedral angles related to the ether functional groups.

The vertical ionization energies of the four conformers increase monotonically from the tetradentate $C_{4}$ conformer to the monodentate $S_{4}$ conformer. Considering the energetic similarity among the first three neutral conformers, this increasing trend is just a direct manifestation of the decreasing interaction strengths between $\mathrm{Na}^{+}$and $12 \mathrm{C} 4$ in the conformation of the neutral forms. These vertical ionization energies correspond to the experimental threshold energies obtained in the present studies. The measured three ionization energies 3.01 $\pm 0.2,3.36 \pm 0.4$, and $3.82 \pm 0.4 \mathrm{eV}$ are in excellent agreement with the predicted vertical ionization energies of the $C_{4}, C_{s}$, and $C_{i}$ conformers $2.99,3.22$, and $3.78 \mathrm{eV}$, respectively. The harmonic vibrational frequency of the sodium ion stretching mode for $\mathrm{Na}^{+}\left(C_{4}\right)$ is $206 \mathrm{~cm}^{-1}$ at the $\mathrm{HF}$ level. The possible steplike features due to the vibrational excitation in the photoionization process were buried in the rising slopes of the photoionization efficiency spectrum.

In the above analysis, the assignments of ionization energies are based on Watanabe plots. The applicability of the method for the diatomic molecules has been supported by an earlier theoretical analysis on the photoionization threshold behavior. ${ }^{31,32}$ For polyatomic molecules, the method has been recommended to be applicable for the molecular systems at high temperature and with low vibrational frequency modes. ${ }^{32,33}$ For the present $\mathrm{Na}(12 \mathrm{C} 4)$ complex, the major nuclear motions involved in photoionization are the sodium stretching and bending motions, and the ring puckering of the crown ether. These motions are all in the low-frequency domain. At the present experimental temperatures, these low-frequency vibrational modes were all densely populated. Apparently, the above high-temperature criterion was met in the present experiments. For the conformers with higher ionization energies, one might expect that the vibrationally induced autoionization would distort the photoionization efficiency intensity in the threshold region. Since the conformers are in the same electronic state, the photoionization transitions would be mainly determined by the Franck-Condon factors between the neutral and ionic electronic states. ${ }^{34}$ This would ensure that direct photoionization of the conformers with lower threshold ionization energies would prevail over the vibrationally induced autoionization process of those with higher threshold ionization energies if the photon energy was below their threshold ionization energies. The absence of any pronounced autoionization structure in the photoionization efficiency spectrum is consistent with this theoretical observation.

In summary, since the $\mathrm{Na}^{+}\left(C_{i}\right)$ conformer is not locally stable and also the possible minor contamination of the ionization signal from the disodium complex was not ruled out in the present experiment, the above threshold ionization energy 
TABLE 4: Theoretical and Experimental Ionization Energies $(\mathrm{eV})$ of $\mathrm{Na}(12 \mathrm{C4})^{a}$

\begin{tabular}{|c|c|c|c|c|c|c|c|c|c|c|}
\hline \multirow[b]{2}{*}{ reaction } & \multicolumn{3}{|c|}{ vertical } & \multicolumn{6}{|c|}{ adiabatic } & \multirow[b]{2}{*}{ exptl } \\
\hline & $\overline{\mathrm{HF}}$ & MP2 & $\overline{\text { B3PW91 }}$ & $\overline{\mathrm{HF}^{b}}$ & $\mathrm{MP}^{b}$ & B3PW91 $^{b}$ & $\mathrm{HF}^{c}$ & $\mathrm{MP}^{c}$ & B3PW91 $^{c}$ & \\
\hline $\mathrm{Na} \rightarrow \mathrm{Na}^{+}$ & & & & 4.94 & 5.00 & 5.27 & & & & $5.139^{d}$ \\
\hline $\mathrm{Na}\left(C_{4}\right) \rightarrow \mathrm{Na}^{+}(12 \mathrm{C} 4)$ & 2.57 & 2.79 & 2.99 & 2.40 & 2.61 & 2.80 & 2.40 & 2.61 & 2.80 & 3.01 \\
\hline $\mathrm{Na}\left(C_{s}\right) \rightarrow \mathrm{Na}^{+}(12 \mathrm{C} 4)$ & 2.80 & 3.01 & 3.22 & 2.39 & 2.60 & 2.81 & 2.54 & 2.75 & 2.95 & 3.36 \\
\hline $\mathrm{Na}\left(C_{i}\right) \rightarrow \mathrm{Na}^{+}(12 \mathrm{C} 4)$ & 3.39 & 3.56 & 3.78 & 2.36 & 2.55 & 2.82 & $e$ & $e$ & $e$ & $3.82^{f}$ \\
\hline $\mathrm{Na}\left(S_{4}\right) \rightarrow \mathrm{Na}^{+}(12 \mathrm{C} 4)$ & 4.01 & 4.15 & 4.39 & 2.23 & 2.39 & 2.69 & 2.74 & 2.96 & 3.18 & \\
\hline
\end{tabular}

${ }^{a}$ At the HF/6-311+G**, MP2(Fu)/6-311+G**, and B3PW91/6-311+G**//HF/6-31G* calculation levels. ${ }^{b}$ Adiabatic equilibrium ionization energy with respect to the most stable conformer $\mathrm{Na}^{+}\left(C_{4}\right) .{ }^{c}$ Adiabatic equilibrium ionization energy in its original conformation state. ${ }^{d}$ Reference 29. ${ }^{e}$ Neutral parent conformation is not locally stable in ionic form. ${ }^{f}$ Estimated value. See text for the details.

TABLE 5: Theoretical Equilibrium Dissociation Energies $(\mathrm{eV})^{a}$

\begin{tabular}{|c|c|c|c|c|c|c|c|c|c|c|c|}
\hline \multirow[b]{2}{*}{ reactions } & \multicolumn{3}{|c|}{ sudden } & \multicolumn{8}{|c|}{ adiabatic } \\
\hline & $\mathrm{HF}$ & MP2 & B3PW91 & $\mathrm{HF}^{b}$ & $\mathrm{MP} 2^{b}$ & B3PW91 ${ }^{b}$ & $\mathrm{ZPEC}^{c}$ & $\mathrm{HF}^{d}$ & $\mathrm{MP} 2^{d}$ & $\mathrm{~B} 3 \mathrm{PW} 91^{d}$ & $\mathrm{ZPEC}^{e}$ \\
\hline \multicolumn{12}{|l|}{ neutral complexes } \\
\hline $\mathrm{Na}\left(C_{4}\right) \rightarrow \mathrm{Na}+12 \mathrm{C} 4$ & 0.589 & 0.586 & 0.487 & 0.209 & 0.239 & 0.166 & -0.037 & 0.508 & 0.527 & 0.401 & -0.046 \\
\hline $\mathrm{Na}\left(C_{s}\right) \rightarrow \mathrm{Na}+12 \mathrm{C} 4$ & 0.423 & 0.450 & 0.378 & 0.197 & 0.225 & 0.183 & -0.031 & 0.337 & 0.376 & 0.296 & -0.041 \\
\hline $\mathrm{Na}\left(C_{i}\right) \rightarrow \mathrm{Na}+12 \mathrm{C} 4$ & 0.346 & 0.376 & 0.318 & 0.188 & 0.237 & 0.205 & -0.031 & 0.264 & 0.329 & 0.274 & -0.043 \\
\hline $\mathrm{Na}\left(S_{4}\right) \rightarrow \mathrm{Na}+12 \mathrm{C} 4$ & 0.097 & 0.146 & 0.104 & 0.068 & 0.128 & 0.092 & -0.019 & 0.068 & 0.128 & 0.092 & -0.019 \\
\hline \multicolumn{12}{|l|}{ ionic complexes ${ }^{f}$} \\
\hline $\mathrm{Na}^{+}\left(C_{4}\right) \rightarrow \mathrm{Na}^{+}+12 \mathrm{C} 4$ & 3.185 & 2.949 & 2.979 & 2.724 & 2.556 & 2.614 & -0.070 & 3.024 & 2.844 & 2.850 & -0.079 \\
\hline $\mathrm{Na}^{+}\left(C_{s}\right) \rightarrow \mathrm{Na}^{+}+12 \mathrm{C} 4$ & 2.984 & 2.756 & 2.805 & 2.572 & 2.382 & 2.472 & -0.062 & 2.711 & 2.534 & 2.584 & -0.072 \\
\hline $\mathrm{Na}^{+}\left(S_{4}\right) \rightarrow \mathrm{Na}^{+}+12 \mathrm{C} 4$ & 2.698 & 2.479 & 2.545 & 2.198 & 1.947 & 2.112 & -0.040 & 2.198 & 1.947 & 2.112 & -0.040 \\
\hline
\end{tabular}

${ }^{a}$ At the $\mathrm{HF} / 6-311+\mathrm{G}^{* *}, \mathrm{MP} 2(\mathrm{Fu}) / 6-311+\mathrm{G}^{* *}$, and B3PW91/6-311+G**//HF/6-31G* calculation levels; all energies were BSSE corrected. ${ }^{b}$ Adiabatic equilibrium dissociation energy with respect to the most stable conformer of 12 -crown- $4, S_{4} .{ }^{c}$ Zero-point energy corrections with respect to the most stable conformer, $S_{4} \cdot{ }^{d}$ Adiabatic equilibrium dissociation energy in its original conformation state. ${ }^{e}$ Zero-point energy corrections in its original conformation state. ${ }^{f} \mathrm{Na}^{+}\left(C_{i}\right)$ is locally unstable.

assigned for the $\mathrm{Na}\left(C_{i}\right)$ conformer was presented here as an estimated value. The other two threshold ionization energies for the $\mathrm{Na}\left(C_{4}\right)$ and $\mathrm{Na}\left(C_{s}\right)$ conformers were free of these uncertainties. A further support of the present conformation assignments which is based on the estimation of the relative ionization intensities of the conformers through the equilibrium bond dissociation energies and conformation geometric symmetry shall be discussed in the following section.

4.F. Equilibrium Bond Dissociation Energies of $\mathrm{Na}(12 \mathrm{C4})$ and $\mathrm{Na}^{+}(\mathbf{1 2 C 4})$. Two types of equilibrium bond dissociation energies were calculated: the sudden equilibrium bond dissociation energies, i.e., the equilibrium bond dissociation energies with the molecular fragment geometries being frozen in the parent geometry, and the adiabatic equilibrium bond dissociation energies in which the molecular fragments are relaxed to the geometries of either the most stable conformer or the locally stable conformer of the parent molecule. The sudden bond dissociation energy serves as a direct measure of the bond interaction strength; the adiabatic bond dissociation energy with respect to the most stable conformer is just the bond dissociation enthalpy at $0 \mathrm{~K}$; and the adiabatic bond dissociation energy in its original parent conformation state, when compared with the sudden bond dissociation energy could be correlated with the extent of the geometric changes of the ligand in the formation of the complex from its locally stable conformer. Here, as a note, the bond dissociation energy is the summation of the equilibrium bond dissociation energy and the corresponding zero-point energy correction.

As shown in Table 5, for the neutral sodium complexes, the sudden dissociation energies are roughly proportional to the number of $\mathrm{Na}-\mathrm{O}$ bonds in each conformer. The linear relation is especially good for the MP2 and B3PW91 results. This suggests that the intrinsic bond strengths of the $\mathrm{Na}-\mathrm{O}$ bonds are more or less additive as the conformation varies from the monodentate to the tetradentate form. Another feature is that the electron correlation effect decreases from the monodentate to tetradentate form if one compares the HF and MP2 energies.
The HF and MP2 sudden dissociation energies of the $C_{4}$ conformer are essentially identical, which suggests that the electron correlation effect is negligible for the tetradentate sudden bond dissociation energy. Along with the general conclusions reached in the subsection of the Mulliken population analysis, these observations are consistent with the finding of Rencsok et al. in their study of $\operatorname{Li}(9 \mathrm{C} 3)_{2}$; that is, the correlation effects are expected to be very small on the physical quantities which are related to the energy eigenstates of an extremely diffusive valence electron. ${ }^{6}$

For the adiabatic equilibrium bond dissociation energies with respect to the most stable $S_{4}$ conformer of $12 \mathrm{C} 4$, the $C_{4}, C_{s}$, and $C_{i}$ bond dissociation energies are essentially the same at the MP2 level; while, at the B3PW91 level, the $C_{i}$ conformer turns out to be the most stable conformer, the $C_{s}$ conformer is the next higher one by $0.022 \mathrm{eV}$, and the following $C_{4}$ conformer is further higher by another $0.017 \mathrm{eV}$. Taking these facts into accounts, one could roughly estimate the population ratio of these three conformers at room temperature as follows. Since the bond dissociation energies and their thermal corrections are close to each for the three most stable conformers, to a good approximation, their thermal equilibrium population ratio would be mainly determined by the ratio of the rotational symmetry numbers of the conformers. ${ }^{35}$ Note that a thermal molecular beam was employed in the present experiments. The rotational symmetry number is 4,1 , and 1 , and the number of the rotationally distinguishable chelating positions is 1,1 , and 2 for the $C_{4}, C_{s}$, and $C_{i}$ conformers, respectively. The resulting population ratio of $C_{4}: C_{s}: C_{i}$ is $1: 4: 8$. The ionization intensity ratio obtained from the photoionization efficiency spectrum is $1: 6: 54$. By assuming that the ionization efficiencies of the three conformers are identical, the ionization intensity ratio would be identical to the population ratio. Apparently, the observed $C_{4}: C_{s}$ population ratio is reasonably in line with the statistical estimation, while the observed $C_{i}$ population is much higher than the statistical value. Several sources could contribute to this discrepancy. One is the possible difference among the bond 
dissociation energies of the conformers, even though their magnitudes may not appear to be that large. For instance, the energy difference between the $C_{4}$ and $C_{i}$ conformer is 0.039 $\mathrm{eV}$ if the B3PW91 energies were taken. At $300 \mathrm{~K}$, their population ratio would increase from 1:8 to $1: 36$. Another contribution may come from the dissociation of the photoionized disodium complex in the $C_{i}$ photoionization wavelength region. ${ }^{28}$ Its contribution has been minimized but was not completely eliminated in the present experiment. Finally, part of the discrepancy may also come from the possible difference in the photoionization efficiencies of the three conformers.

For the neutral conformers, the adiabatic equilibrium bond dissociation energies in the initial parent conformation, as expected, are all lower than the corresponding sudden equilibrium bond dissociation energies by less than $0.069 \mathrm{eV}$ at the MP2 level. This is consistent with the above observation that the comparatively weak $\mathrm{Na}-\mathrm{O}$ bond does not perturb the ligand geometries much during the formation of the complexes.

In the case of the cationic conformers, as shown in Table 5, the following features are observed: (a) the MP2 energies are in good agreement with the corresponding B3PW91 energies, particularly in the sudden dissociation energies; (b) the HF energies are consistently higher than the corresponding MP2 and B3PW91 energies by an average of $0.195 \mathrm{eV}$; (c) due to the much larger interaction energy between the sodium ion and the ether functional group than the energy differences among the conformers of the free crown ether, the relative bond dissociation energies of the three locally stable cationic conformers $-C_{4}, C_{s}$, and $S_{4}$-are dominated by the former interactions and, hence, consistent with the conventional stability picture of the cationic conformers; that is, the tetradentate $C_{4}$ form is the most stable, while the monodentate $S_{4}$ form is the least stable conformer; (d) the largest bonding energy variation comes from the sudden and adiabatic bond dissociation energies in the $S_{4}$ conformer. These observations also suggest that the contribution of the electron correlation to the bond energy is not negligible, and the strong electrostatic interactions between the sodium cation and the four ether functional groups dominate the complex such that the ligand structure was usually strongly distorted to accommodate the formation of the $\mathrm{Na}^{+}-\mathrm{O}$ bonds. The latter case happens, for instance, in the $S_{4}$ conformer, in which essentially four $\mathrm{Na}^{+}-\mathrm{O}$ bonds were formed and the corresponding ligand conformation is significantly distorted from that of the free state.

Experimentally, it has been recommended that the bond dissociation energy of $\mathrm{Na}^{+}(12 \mathrm{C} 4)$ with respect to the most stable conformer at $0 \mathrm{~K}$ is $2.61 \pm 0.13 \mathrm{eV} .{ }^{16}$ The present theoretical values of 2.654 (HF), 2.486 (MP2), and 2.544 (B3PW91) eV are all in agreement with the experimental result to within the cited experimental uncertainty. Since the present experimental threshold ionization energy of the $C_{4}$ conformer corresponds to the theoretical vertical ionization energy, along with the experimental ionization energy of the sodium atom, one may use the energy conservation relation to check the internal consistence of the theoretical neutral and ionic bond dissociation energy among the present three calculation levels. Restricting the following consideration of the states to the $C_{4}$ conformation, the energy conservation relation between the adiabatic equilibrium bond dissociation energies $D_{\mathrm{e}}$ 's and the adiabatic equilibrium ionization energies $I_{\mathrm{a}}$ 's is

$$
I_{\mathrm{a}}\left(\mathrm{Na}\left(C_{4}\right)\right)+D_{\mathrm{e}}\left(\mathrm{Na}^{+}\left(C_{4}\right)\right)=I_{\mathrm{a}}(\mathrm{Na})+D_{\mathrm{e}}\left(\mathrm{Na}\left(C_{4}\right)\right)
$$

With the help of the theoretical energy difference between the vertical and adiabatic ionization energies and the corresponding zero-point energy corrections listed in Tables 4 and
5 , the experimental adiabatic equilibrium ionization energy of $I_{\mathrm{a}}\left(\mathrm{Na}\left(C_{4}\right)\right)$ is $2.87 \mathrm{eV}$. The sodium atom ionization energy is $5.139 \mathrm{eV} .{ }^{29}$ Substituting the theoretical $D_{\mathrm{e}}\left(\mathrm{Na}^{+}\left(C_{4}\right)\right)$ values of 3.024 (HF), 2.844 (MP2), and 2.850 (B3PW91) eV into the above equation, one obtains the corresponding predicted $D_{\mathrm{e}^{-}}$ $\left(\mathrm{Na}\left(C_{4}\right)\right)$ values of $0.755,0.573$, and $0.578 \mathrm{eV}$, respectively. Compared with the theoretical values of $0.508,0.527$, and 0.401 $\mathrm{eV}$, the results of the MP2 level have the best internal energy consistence with respect to the available experimental results. The values of the HF and B3PW91 levels are off by 0.247 and $0.177 \mathrm{eV}$, respectively. These discrepancies are all large enough to be ruled out by the present experimental results. In short, for the neutral and ionic bond dissociation energies, the MP2 calculations yield more consistent results than those by the HF and B3PW91 calculation methods. This conclusion is consistent with the energetic results of Feller and co-workers on the complexes of alkali cations with simple and complex ethers. ${ }^{15}$ Note that for the present complex systems the energetic discrepancies calculated by the B3LYP method, which are not specifically considered here, are close to those of the HF method.

At room temperature, it has been shown by Armentrout and co-workers that in the gas phase the $\mathrm{Na}^{+}(12 \mathrm{C} 4)$ complex is mainly in the $C_{4}$ conformation. ${ }^{16}$ This specificity of the $C_{4}$ configuration is attributed to the extra energetic stability through the full interaction between the cation and the tetradentate $12 \mathrm{C} 4$ ligand. ${ }^{16,36}$ In contrast to the cation complexes, the neutral sodium complex is mainly in the $C_{i}$ conformation instead of the $C_{4}$ one. The present analysis suggests that the conformer entropy instead of the conformer energy is the major factor in determining the relative populations of the neutral $C_{4}, C_{s}$, and $C_{i}$ conformers.

\section{Conclusions}

In the present paper, the conformation-dependent properties of the $\mathrm{Na}(12 \mathrm{C} 4)$ and $\mathrm{Na}^{+}(12 \mathrm{C} 4)$ molecular complexes were reported. With the help of the theoretical ionization energies, the general features of the ionization efficiency spectra of $\mathrm{Na}$ (12C4) were assigned as the contribution from the various conformers, and their experimental threshold ionization energies were obtained. The availability of the experimental ionization energies also enables one to assess the internal energy consistency of the theoretical neutral and ionic bond dissociation energies among the various ab initio calculation methods. The relative photoionization intensities as shown in the photoionization efficiency spectrum suggest that the $C_{i}$ conformer is the dominant species under the present experiment condition. It is suggested that the conformer entropy instead of the energy is the major factor in determining the relative conformer population for this neutral system. This is in contrast to the preference of the $C_{4}$ conformer in the corresponding cationic complex as observed by Armentrout and co-workers.

With the Mulliken electron population analysis, the spatial distribution of the valence electron of the complex was found to vary from the Na-localized valence-type to the diffusivetype distribution as the conformation varies from the monodentate to the tetradentate form. Under the condition that the adiabatic conformation energies are more or less equivalent, it is expected that the chemical properties of the complex are also going to be conformation-dependent. These related subjects are interesting and deserve further investigation.

Acknowledgment. The financial support of this work by the National Science Council, Taiwan, R.O.C., is gratefully acknowledged. 
Supporting Information Available: Tables of the geometric parameters and harmonic vibrational frequencies of the stable conformers of $12 \mathrm{C} 4, \mathrm{Na}(12 \mathrm{C} 4)$, and $\mathrm{Na}^{+}(12 \mathrm{C} 4)$ discussed in this paper ( 9 pages). Ordering information is given on any current masthead page.

\section{References and Notes} 271

(1) Dye, J. L.; Debacker, M. G. Аnпu. Rev. Phys. Chem. 1987, 38 ,

(2) Wagner, M. J.; Dye, J. L. Annu. Rev. Mater. Sci. 1993, 23, 223.

(3) Dye, J. L. Chemtracts; Inorg. Chem. 1993, 5, 243.

(4) Allan, G.; DeBacker, M. G.; Lannoo, M.; Lefebvre, J. Europhys. Lett. 1990, 11, 49 .

(5) Rencsok, R.; Kaplan, T. A.; Harrison, J. F. J. Chem. Phys. 1990, 93, 5875 .

(6) Rencsok, R.; Kaplan, T. A.; Harrison, J. F. J. Chem. Phys. 1993, 98, 9758.

(7) Singh, D. J.; Krakauer, H.; Haas, C.; Pickett, W. E. Nature 1993, 365,39

(8) Dye, J. L.;Wagner, M. J.; Overney, G.; Huang, R. H.; Nagy, T. F.; Tomanek, D. J. Am. Chem. Soc. 1996, 118, 7329.

(9) Liau, Y.-H.; Su, T.-M. J. Am. Chem. Soc. 1992, 114, 9169.

(10) Hsu, C.-L.; Yeh, T.-S.; Su, T.-M. J. Phys. Chem. 1994, 98, 8876

(11) Yeh, T.-S.; Su, T.-M. J. Phys. Chem. A 1997, 101, 1672.

(12) Katritzky, A. R.; Malhotra, N.; Ramanathan, R.; Kemerait, R. C.; Zimmerman, J. A.; Eyler, J. R. Rapid Commun. Mass Spectrosc. 1992, 6 , 25.

(13) Glendening, E. D.; Feller, D.; Thompson, M. A. J. Am. Chem. Soc. 1994, 116, 10657

(14) Ray, D.; Feller, D.; More, M. B.; Glendening, E. D.; Armentrout,

P. B. J. Phys. Chem. 1996, 100, 16116.

(15) Feller, D.; Apra, E.; Nichols, J. A.; Bernholdt, D. E. J. Chem. Phys. 1996, $105,1940$.

(16) More, M. B.; Ray, D.; Armentrout, P. B. J. Phys. Chem. A 1997, 101,831 .

(17) Kuan, T.-C.; Jiang, R. C.; Su, T.-M. J. Chem. Phys. 1990, 92, 2553.

(18) Frisch, M. J.; Trucks, G. W.; Schlegel, H. B.; Gill, P. M. W.; Johnson, B. G.; Robb, M. A.; Cheeseman, J. R.; Keith, T.; Petersson, G.
A.; Montgomery, J. A.; Raghavachari, K.; Al-Laham, M. A.; Zakrzewski, V. G.; Ortiz, J. V.; Foresman, J. B.; Cioslowski, J.; Stefanov, B. B.; Nanayakkara, A.; Challacombe, M.; Peng, C. Y.; Ayala, P. Y.; Chen, W.; Wong, M. W.; Andres, J. L.; Replogle, E. S.; Gomperts, R.; Martin, R. L.; Fox, D. J.; Binkley, J. S.; Defrees, D. J.; Baker, J.; Stewart, J. P.; HeadGordon, M.; Gonzalez, C.; Pople, J. A. Gaussian 94, Revision C.3; Gaussian, Inc.: Pittsburgh, PA, 1995

(19) Seidl, E. T.; Schaefer, H. F. J. Phys. Chem. 1991, 95, 3589.

(20) Murcko, M. A.; DiPaola, R. A. J. Am. Chem. Soc. 1992, 114, 10010.

(21) Hashimoto, K.; Morokuma, K. J. Am. Chem. Soc. 1994, 116, 11436.

(22) Hashimoto, K.; Morokuma, K. J. Am. Chem. Soc. 1995, 117, 4151

(23) Nicely, V. A.; Dye, J. L. J. Chem. Phys. 1970, 52, 4795.

(24) Trenary, M.; Schaefer, H. F., III; Kollman, P. A. J. Am. Chem. Soc. 1977, 99, 3885.

(25) Bentley, J. J. Am. Chem. Soc. 1982, 104, 2754

(26) Curtiss, L. A.; Kraka, E.; Gauss, J.; Cremer, D. J. Phys. Chem 1987, 91, 1080

(27) Hsiao, Y.-W.; Chang, K.-M.; Su, T.-M. Chem. Phys. 1992, 162, 335.

(28) The theoretical adiabatic ionization energy of $\mathrm{Na}_{2}\left(C_{4}\right)$ is $3.39 \mathrm{eV}$. The bond dissociation energy of $\mathrm{Na}_{2}+\left(C_{4}\right) \rightarrow \mathrm{Na}+\mathrm{Na}^{+}\left(C_{4}\right)$ is $0.27 \mathrm{eV}$ The threshold photoionization energy for the sodium atom to split from $\mathrm{Na}_{2}{ }^{+}\left(C_{4}\right)$ is estimated to be $3.66 \mathrm{eV}$ or $339 \mathrm{~nm}$ photon.

(29) Moore, C. E. Atomic Energy Levels; NSRD-NBS Vol.1; US GPO Washington, DC, 1971; p 89

(30) Martin, S.; Chevaleyre, J.; Valignat, S.; Perrot, J.; Broyer, M.; Cabaud, B.; Hoareau, A. Chem. Phys. Lett. 1982, 87, 235.

(31) Guoyn, P. M.; Berkowitz, J. J. Chem. Phys. 1971, 54, 1814.

(32) Berkowitz, J. In Advances in High-Temperature Chemistry; Eyring, L., Ed.; Academic Press: New York, 1971; Vol. 3, p 123.

(33) Dao, P. D.; Peterson, K. I.; Castleman, A. W., Jr. J. Chem. Phys. 1984, 80, 563.

(34) Berkowitz, J. Photoabsorption, Photoionization, and Photoelectron Spectroscopy; Academic Press: New York, 1979.

(35) McQuarrie, D. A. Statistical Mechanics; Harper \& Row Publishers: New York, 1976; p 142.

(36) More, M. B.; Ray, D.; Armentrout, P. B. J. Phys. Chem. A 1997, $101,7007$. 